Questions vives

\section{Questions Vives}

Recherches en éducation

Vol.9 $n^{\circ} 19$ | 2013

Éducation routière, changement de comportement et formation à la conduite : constat, enjeux et transformations

\title{
Vers une éducation à la prudence : délibérer dans l'incertitude. Le cas de l'usage de la voie publique
}

Education geared towards caution: deliberate in uncertainty. The case of the use of public roads

\section{Dominique Violet}

\section{(2) OpenEdition}

1 Journals

\section{Édition électronique}

URL : http://journals.openedition.org/questionsvives/1291

DOI : 10.4000/questionsvives.1291

ISBN : 978-2-8218-1394-6

ISSN : $1775-433 \mathrm{X}$

Éditeur

Université Aix-Marseille (AMU)

Édition imprimée

Date de publication : 15 juillet 2013

Pagination : 107-122

ISBN : 978-2-912643-43-8

ISSN : $1635-4079$

Référence électronique

Dominique Violet, « Vers une éducation à la prudence : délibérer dans l'incertitude. Le cas de l'usage de la voie publique », Questions Vives [En ligne], Vol. 9 n¹9 | 2013, mis en ligne le 15 octobre 2013, consulté le 02 mai 2019. URL : http://journals.openedition.org/questionsvives/1291 ; DOI : 10.4000/ questionsvives.1291

Ce document a été généré automatiquement le 2 mai 2019.

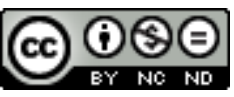

Questions Vives est mis à disposition selon les termes de la licence Creative Commons Attribution -

Pas d'Utilisation Commerciale - Pas de Modification 4.0 International. 


\section{Vers une éducation à la prudence : délibérer dans l'incertitude. Le cas de l'usage de la voie publique}

Education geared towards caution: deliberate in uncertainty. The case of the use of public roads

Dominique Violet

\section{Introduction}

1 L'usage raisonné de la voie publique et donc la prévention des accidents reste un problème prégnant de notre société. Si le dispositif répressif mis en œuvre a nettement fait baisser le nombre de morts sur les routes françaises, on constate depuis 2007 une tendance à la stabilisation (diminution de 1,9\% du nombre de tués et augmentation de $1,1 \%$ du nombre de blessés, les spécialistes parlent d'effet plateau). On se plait alors à rêver que la recherche technologique fournira, peut-être, dans un futur proche, la réponse pour une sécurité absolue. Découverte d'un Big Brother susceptible de voir chaque conducteur, à tout moment et en toutes circonstances, directement inspiré du Meilleur des mondes. Ou encore, version édulcorée de la précédente, mise au point d'un système panoptique tel qu'imaginé par Bentham où on laisse à penser à chaque usager qu'il est, à tout moment et en toutes circonstances, surveillé, sans qu'il lui soit possible de savoir si cela est vrai. On peut enfin, fantasmer, comme dans d'autres œuvres de science-fiction, à la mise sur le marché de véhicules auxquels il suffit de communiquer le lieu où l'on souhaite se rendre et dont les calculateurs prennent en compte tous les paramètres pour définir le trajet et assurer une sécurité sans faille.

2 Ces différents procédés n'étant aujourd'hui que des anticipations hasardeuses, il convient donc de s'efforcer de policer le conducteur à l'aide des deux leviers habituels, la répression et l'éducation. Les efforts de la Délégation interministérielle à la sécurité 
routière concernent surtout l'activation du deuxième levier afin d'atteindre les objectifs européens cohérents avec la matrice GDE. C'est dans cet esprit d'éducation que s'inscrivent nos travaux de recherche.

3 Nous commencerons par appréhender la voie publique comme une organisation complexe qui contient des « zones d'incertitude » permettant à chaque acteur de disposer d'un certain degré de liberté afin d'élaborer des choix pour se déplacer. Ensuite nous nous efforcerons de modéliser le processus de délibération conduisant à un choix éthique. Pour cela nous nous réfèrerons notamment à la notion de prudence esquissée par Aristote. Enfin nous présenterons une investigation empirique dont les résultats permettent d'entrevoir la nécessité d'un projet d'éducation à la prudence pour un usage raisonné de la voie publique.

\section{La voie publique : une organisation avec « zone d'incertitude »}

4 La voie publique est l'espace au sein duquel chacun peut accomplir les déplacements qu'il a choisis ou qui lui sont imposés, quels que soient le moment et le mode retenus, sans gêner et sans être gêné par les autres, sans être cause de danger pour les autres ou être mis en situation de danger de leur fait. On peut concevoir cet espace comme une organisation, et partant, dériver certaines notions fécondes pour l'analyse des organisations à l'étude de la voie publique.

5 La voie publique peut être assimilée à une organisation dans la mesure ou : on y affiche un ou des objectifs, on dispose d'un système de communication, on utilise des outils, on met en scène des acteurs dotés de tâches, on régule les interactions acteurs/outils/ acteurs.

6 L'objectif de l'organisation "voie publique » est de faire en sorte que chacun de ses usagers puisse se rendre où il veut, quand il l'a décidé, en utilisant le moyen de déplacement qu'il souhaite au sein d'une collection de moyens autorisés et compatibles, sans causer de gêne ou dommages aux autres usagers et à leurs biens. La communication utilisée sur la voie publique est assurée par des signaux, généralement visuels (parfois sonores), dont la signification est standardisée et inscrite dans le Code de la route. Chaque signal a une signification qui informe l'usager sur la conduite à tenir (ralentir, changer de file, ne pas doubler, etc.)

7 Les objets caractéristiques de cette organisation correspondent à deux catégories de fonction : l'utilisation ou la régulation. L'utilisation englobe les moyens de locomotion et les accessoires qui s'y rattachent pour améliorer la sécurité (gilet ou brassard fluorescent pour être mieux vu, casque pour protéger la tête, etc.) et le confort des usagers (ustensiles divers tels que GPS, lunettes de soleil, radio, etc.). Des machines ou dispositifs contribuent à la régulation des déplacements (feux tricolores, chaussées, trottoirs, panneaux divers, etc. En ce qui concerne les personnes, on peut également les classer dans deux catégories : les usagers qui utilisent un moyen de déplacement (automobilistes, cyclistes, piétons, etc.); les agents œuvrant à la régulation du trafic: policiers, gendarmes, personnels d'entretien des voies et des chaussées. Il convient de remarquer que ces deux catégories sont poreuses. D'une part, l'usager joue un rôle dans la régulation, en écoutant et suivant, par exemple les conseils de Bison futé ou en décalant son heure d'embauche grâce à une répartition adéquate de son temps de travail ou encore en utilisant l'autobus 
au lieu de sa voiture personnelle. D'autre part, l'agent de régulation est aussi un usager, y compris parfois dans l'exercice de ses fonctions (policier qui patrouille, personnel d'entretien des chaussées qui transporte des matériaux, etc.).

8 Les interactions acteurs/outils ou acteurs/acteurs ou acteurs/acteurs avec médiation par des outils sont définies par des répertoires de spécifications (code de la route, inventaire des compétences minimales pour les usagers, instructions diverses pour les agents du trafic, normes imposées par les Mines pour donner droit à circulation à un véhicule, etc. tâche de l'usager consiste à définir et emprunter un trajet pour se rendre en un lieu déterminé, au moment choisi, dans un temps généralement limité, sans se blesser ou blesser autrui et sans entraver la circulation pour les autres usagers. La tâche de l'agent de régulation visera à faciliter et sécuriser la circulation des usagers. Il est à noter que les activités des différents acteurs sont accordées entre elles par le biais des procédures (Code de la route). Ainsi, un feu rouge est couplé à un feu vert afin que lorsqu'un acteur s'arrête, celui qui croise son chemin peut passer sans causer de troubles. En termes de Système de contribution rétribution, l'usage de la voie publique exige l'adhésion de l'usager aux règles édictées, la rétribution se traduisant en termes de sécurité, de fluidité, de moindre consommation et pollution.

Cet inventaire s'inspire de Bernoux (1985, pp. 118-123) qui distingue les traits suivants : division des tâches, distribution des rôles, système d'autorité, système de communication, système de contribution-rétribution. Nous y avons ajouté les objectifs de l'organisation.

Considérer la voie publique comme une organisation permet, pour en faire l'étude, de recourir à certaines notions auxquelles on peut accorder une réelle valeur heuristique. Ainsi, il est possible d'accorder à l'usager un statut d'acteur et d'utiliser certaines constructions théoriques permettant de déchiffrer ses comportements.

Pour Crozier et Friedberg (1977), c'est à partir de la compréhension que les acteurs ont de l'organisation qui les emploie qu'ils développent des stratégies propres à atteindre leurs buts dans les conditions qu'ils perçoivent comme les plus favorables pour eux. Ainsi, la notion de « logique d'action » caractérise le mode opératoire de l'acteur, conçu et mise en œuvre à partir d'une analyse et d'une évaluation des possibilités offertes par l'organisation pour parvenir à ses fins. Cette construction de logiques personnelles d'action n'est possible que parce que toute organisation offre à chacun des acteurs concernés une marge de liberté liée à des zones d'incertitude. Cette notion, cardinale dans la construction théorique de Crozier et Friedberg, désigne l'espace où le règlement ne contraint pas l'acteur et où il peut donc agir, dans une certaine mesure, à sa guise, devenant de la sorte imprévisible. Cette absence de contrainte et donc cette liberté concédée lui permet de négocier sa coopération.

Cette notion de zone d'incertitude peut paraître pertinente pour l'analyse, par exemple, du comportement de l'automobiliste, la fréquentation quotidienne de la voie publique montrant que la conduite n'est pas toujours régie par les préconisations du code de la route. L'usage de la voie publique procure ainsi des espaces de liberté permettant aux acteurs de faire des choix et d'agir comme ils l'entendent quant à la conduite à tenir. Ainsi, l'automobiliste peut choisir de prendre la rue $X$ plutôt que la rue $Y$, de rouler en $4^{\mathrm{e}}$ plutôt qu'en $5^{\mathrm{e}}$, etc. Ces marges de liberté peuvent toucher aussi des comportements proscrits, par exemple rouler à gauche. Il est à noter que certains comportements non interdits par le Code de la route peuvent s'avérer très dangereux (il n'est pas interdit de prendre le volant épuisé mais seulement déconseillé). À l'inverse, certains comportements interdits n'accroissent pas les dangers de la circulation (les spécialistes 
estiment que les conducteurs sans permis de conduire valide causent moins d'accidents que les conducteurs en possession de permis en règle).

Les zones d'incertitude, c'est-à-dire les lieux et les moments au cours desquels l'automobiliste peuvent faire des choix peuvent se définir selon deux critères : absence ou invisibilité des représentants de l'ordre; absence d'obstacles à la mise en œuvre de certaines manœuvres ou comportements. Plus globalement, les zones d'incertitudes se définissent comme l'espace-temps dans lequel on ne sait pas ce qui va se passer. Les acteurs sont libres d'agir à leur guise ou n'ont pas appris à faire face à l'événement qui advient. Ainsi l'incertitude se présente comme une crise, une rupture à dans une organisation. C'est donc de l'incertitude que procède le risque.

14 Selon le cadre épistémologique auquel on adhère, le risque revêt des acceptions différentes. Si l'on adhère au paradigme prométhéen de la sécurité, le risque est assimilé à un vecteur orienté vers une direction négative.

\section{Choix éthique}

Il conviendra de noter que tout usage de la voie publique est précédé d'un projet d'usage, plus ou moins conscient, établi par l'usager. Cette notion est importante notamment en matière de formation. Ainsi, le manquement à des règles édictées par le Code de la route, peut être programmé dès le projet, sans que l'usager se confronte à des aléas le conduisant à prendre, dans l'urgence, une décision aboutissant à un comportement illicite. Il en est ainsi lorsqu'un usager, par exemple, se voyant en retard pour se rendre à son travail, décide, non d'accepter son retard et de le reporter sur son heure d'entrée mais de chercher à le compenser en dépassant les vitesses autorisées.

Une façon habituelle d'appréhender l'usager de la voie publique est de le saisir comme un acteur dont la conduite est orientée par un respect absolu du code de la route. Dès qu'il se trouve sur la voie publique, l'usager est en recherche de conformité avec les règles en usage. Cette figure de l'usager, nous la nommerons "opérateur qui s'accommode». Le terme opérateur revêt le sens que lui attribuent les ergonomes, c'est-à-dire celui qui, par les comportements qu'il développe, s'efforce de réaliser la tâche attendue de lui à travers des procédures prescrites. Le verbe "S'accommoder» est pris au sens que Piaget lui donne dans ses constructions théoriques, c'est-à-dire qu'il caractérise l'effort que déploie le sujet pour répondre aux commandes de son environnement. L'usager de la voie publique, appréhendé sous cet angle, détermine son trajet et ajuste ses actes en fonction des préconisations prodiguées, il se moule dans le désir des régulateurs des flux de la circulation. Un tel protagoniste n'est pas forcément dépourvu d'autonomie, mais il est perçu comme un acteur rationnel ayant conscience que son intérêt ne peut se réaliser que par l'adhésion aux normes en vigueur.

En changeant de référence théorique et en se tournant vers la sociologie de Boltanski et Thévenot (1991), on pourrait voir dans cette recherche de conformité au code de la route, l'attrait et la poursuite d'une grandeur de type de civique par des acteurs vivant dans un monde directement dérivé de la Cité civique, univers totalement spéculatif où le principe supérieur est celui de la subordination de la volonté individuelle à la volonté générale. L'usager devient un acteur désincarné, la grandeur qu'il recherche est le respect absolu de règles reposant sur une forme de contrat social. Ce monde simple et fictif, ordonné par 
un principe supérieur unique et non-sujet à débat, permet d'établir des hiérarchies entre les acteurs, de repérer et qualifier les violations.

\subsection{De la défaillance au choix}

18 Toutefois, le monde réel n'est pas un simple dérivé d'une cité à principe unique et incontestable, il est de nature composite, c'est-à-dire animé par des principes qui coexistent et s'accordent entre eux ou s'entrechoquent. Dans un tel univers, le Code de la route n'est plus le principe absolu, il cohabite avec d'autres principes qui agissent sur le comportement des acteurs. L'usager peut alors être saisi différemment d'un opérateur obéissant à un principe hégémonique. On peut voir en lui un individu qui interprète et conteste un Code de la route passé au crible d'autres principes auxquels il peut contrevenir et qui lui sont jugés supérieurs. Ainsi, l'usager, dans sa pratique quotidienne de la voie publique, peut-être conduit à affronter des situations qui mettent en concurrence le code de la route comme principe avec d'autres tels la solidarité, l'assistance à quelqu'un, l'hédonisme, etc. Il peut, dès lors, être appréhendé comme un sujet qui opère consciemment et en toute connaissance de cause, des choix qu'il est prêt, le cas échéant, à assumer. Émerge ainsi, pour caractériser l'usager de la voie publique, la figure d'une personne qui délibère, c'est-à-dire qui récapitule des principes, procède à des tris pour ne retenir que ceux qui sont pertinents eu égard à la situation, établit des hiérarchisations entre ceux qui peuvent se trouver en compétition, arrête un choix et le met en œuvre. Cette figure complexifie notablement le travail des analystes de l'accidentologie, l'erreur n'est plus seulement une règle oubliée ou mal assimilée, une mauvaise appréciation de distance, un réflexe émoussé, mais un choix dont il conviendra de s'appliquer à connaître les mécanismes pour améliorer la sécurité.

Concevoir l'usager comme une personne qui décide n'est pas valable en toute circonstance. Pour de nombreuses activités humaines, une partie non négligeable des décisions et des actes s'effectue sans prise de conscience, l'action est pilotée par des routines qui échappent à l'attention de l'acteur. Par ailleurs, introduire la notion de choix, n'est pas davantage une démarche destinée à fonder une entreprise juridico morale de la responsabilité de l'usager. Il ne s'agit que de proposer, au plan cognitif, une explication plausible à certains de ses comportements.

Ce qui est important dans ces modèles en concurrence, c'est l'interprétation qui sera fournie pour expliquer les manquements. D'une part, si l'on se réfère à la figure de "l'opérateur qui s'accommode", les manquements trouvent leur explication dans un postulat de la défaillance, caractéristique d'un acteur connaissant une faiblesse momentanée. D'autre part, si l'on s'adhère à la figure de « la personne qui délibère » les manquements sont expliqués par un postulat du choix, caractéristique d'une décision prise par un sujet après avoir effectué un calcul.

21 Dans un postulat de la défaillance, les manquements sont le produit de situations où l'usager est débordé par les circonstances. On relève (une ou) des altérations en un ou plusieurs points du système usager-véhicule-voie publique qui conduisent à une faute de conduite. On peut, de manière non exhaustive, citer les défaillances suivantes: inattention, fatigue, temps de réaction, consommation d'alcool ou de stupéfiants, etc. 


\subsection{Variété des choix}

Assimilée à une organisation, la voie publique contient des zones d'incertitude laissant des choix aux acteurs. Si chaque usager de la voie publique dispose d'une zone de liberté, cela implique qu'il est libre d'effectuer des choix dans la façon de s'acquitter de sa tâche.

Le conducteur est donc amené à effectuer un nombre considérable de choix dont la majeure partie est probablement non consciente. Il est à noter qu'il n'existe pas d'actes appartenant en propre à la catégorie conscient ou non conscient, cela dépend des circonstances. Mettre le clignotant est généralement un acte non conscient, mais il peut devenir conscient et même donner lieu à stratégie si, par exemple, un automobiliste est suivi d'un camion roulant très prêt, le clignotant sera déclenché très en avance pour être sûr qu'il soit perçu.

Le postulat de la défaillance pose que les choix conscients sont en conformité avec le code de la route et que seuls certains choix non conscients peuvent être illicites. Ainsi, nombre de conducteurs verbalisés au-dessus d'une vitesse autorisée n'ont pas délibérément fait fi d'une interdiction, mais ont plutôt manifesté un défaut d'attention, le manquement n'est pas volontaire.

Il convient, à présent de s'interroger sur la nature des choix qu'opèrent les utilisateurs de la voie publique. On peut qualifier de techniques les choix concernant les moyens utilisés pour réaliser une tâche. Ainsi, si j'ai décidé de me rendre rue $\mathrm{X}$ à telle heure et qu'aucune considération parasite n'entre en compte (par exemple économiser du carburant ou éviter de contribuer à la pollution de la cité, etc.) le choix du moyen de transport, l'itinéraire retenu, le lieu de stationnement constituent des choix techniques.

26 Néanmoins, il est aussi des moments où l'usager est contraint d'effectuer des choix qui mettent en jeu plusieurs principes fondamentaux auxquels il adhère et qui dans certains cas peuvent s'avérer inconciliables. Ainsi, si être à l'heure et respecter les vitesses autorisées sont deux principes auxquels de nombreux usagers adhèrent, et qui sont généralement couplés sans problème, il n'en est plus de même dans le cas d'un retard. Il convient alors d'opérer un choix entre ces deux principes mis en compétition. On est alors dans le cadre d'un choix éthique qu'il convient d'opérer préalablement à tout choix technique. Trier, hiérarchiser des valeurs, sont les opérations mises en œuvre dans le cadre d'un choix éthique.

27 Dans le cadre du postulat du choix, non seulement l'usager effectue des choix techniques mais aussi des choix que l'on qualifiera d'éthiques dans la mesure ou plusieurs principes ou valeurs sont mis en concurrence. C'est ce qui fait la différence avec le postulat de la défaillance au sein de laquelle on ne prête à l'usager qu'un principe unique, celui du respect du code de la route (le non-respect n'est pas analysé comme un rejet à un moment donné d'un élément du code parce qu'il entrerait en compétition avec autre chose mais comme une défaillance), il n'opère ainsi que des choix techniques.

\subsection{Modélisation du processus de délibération}

C'est en revisitant l'Éthique à Nicomaque d'Aristote que Ricoeur propose de différencier Ethique et Morale : «c'est donc par convention que je réserverai le terme d'éthique pour la visée d'une vie accomplie et celui de morale pour l'articulation de cette visée dans des 
normes caractérisées à la fois par la prétention à l'universalité et par un effet de contrainte », écrit Ricoeur.(1990).

La visée éthique ou "visée de la vie bonne avec et pour autrui dans des institutions justes » est première par rapport à la morale et à ses normes. Une vie accomplie est la fin ultime de l'action humaine, quelle que soit l'image que chacun se fait de l'image d'une vie accomplie. Pour autant, la visée éthique ne saurait se dispenser d'un passage par le crible de la norme. En effet, pour savoir si une vie a été bonne, il convient de savoir ce qui est bon. Il faut ainsi recourir à la morale qui, dans l'ordre du bien et du mal se rapporte à des lois, des normes, des impératifs, des interdits caractérisés par l'universalité et l'absolu.

Si éthique et morale sont complémentaires, il est aussi des cas où le caractère universel et absolu des normes devient inapproprié et conduit à des situations conflictuelles que Ricoeur nomme " apories du devoir ». Il revient alors au sujet de formuler des jugements moraux en situation qui renvoient à l'intuition initiale de l'éthique de vie accomplie. Ces jugements moraux en situation de conflits de normes, font appel à des délibérations set recherchent un «juste milieu» qui pour autant ne saurait être réduit à un lâche compromis.

Le « juste milieu est ce troisième moment de la réflexion éthique de Ricoeur que nous essayerons d'accommoder à l'utilisation de la voie publique. L'usager de la voie publique ne peut dans tous les cas être un simple «applicateur» du code de la route. Si effectivement le code de la route n'entre pas en conflit avec d'autres sources de régulation des conduites, il peut, dans certaines situations être mis en concurrence. Soumis à des prescriptions qui ont chacune leur légitimité, l'usager doit se livrer à un calcul, une délibération en situation qui le conduira à arrêter une action qui s'apparente à la sagesse pratique, retour de la visée éthique permettant de conserver l'estime de soi.

À partir des travaux de Ricoeur (1983, 1990), l'on peut appréhender l'éthique du conducteur automobile comme la recherche d'un « juste milieu » entre quatre ordres de préconisations qui peuvent être en tension.

Les travaux de Ricœur sur l'éthique nous ont conduit à retenir un système, possiblement en tension, de quatre types de repères, tels que figuré dans le schéma suivant :

Schéma $1: 4$ types de repères

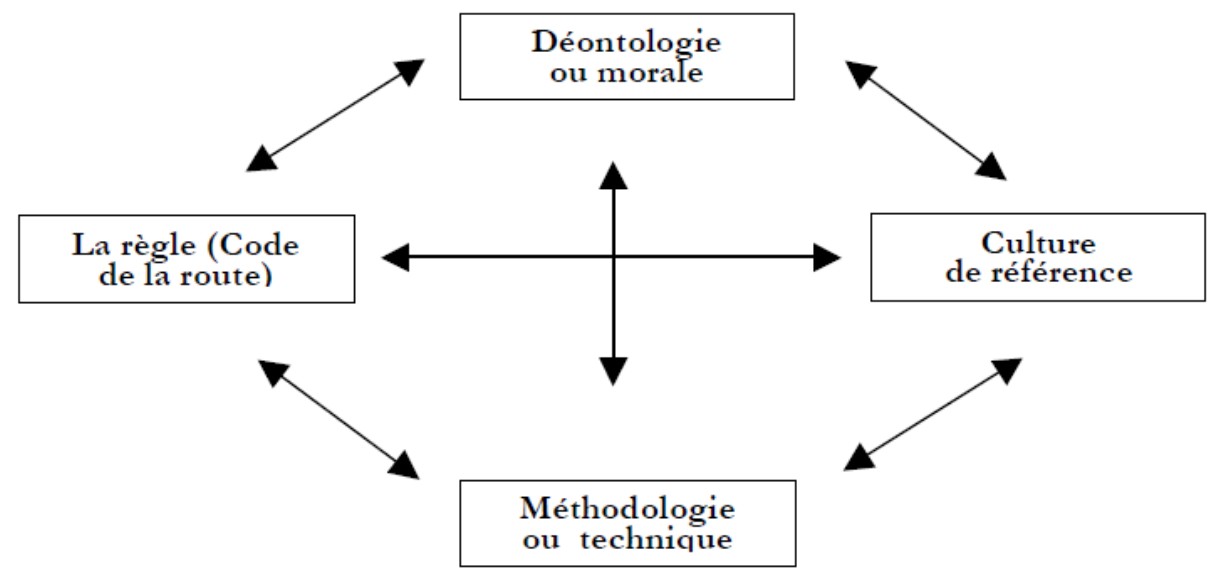



du groupe d'appartenance ou du groupe de référence. Les usages de la voie publique donnent lieu à des styles qui ne se réduisent pas seulement à des techniques mais qui empruntent aussi à des interprétations particulières du code de la route (déni de certaines obligations ou interdits) ou de la morale (rejet de certains commandements, insensibilité à l'autre, hédonisme forcené, etc.).

\subsection{Consonance et dissonance}

38 Les messages prodigués par chacun des ordres considérés peuvent aller dans le même sens et nous parlerons de consonance ou, au contraire, s'avérer discordants, nous parlerons de dissonance.

Exemple de convergence des quatre repères - Dans un grand nombre de situations, le Code de la route peut suffire à réguler la conduite de l'usager sur la voie publique parce qu'il est en congruence avec les autres sources de prescriptions. S'arrêter au feu rouge est une prescription du code de la route qui peut être en accord avec d'autres prescriptions telle que la valeur morale "respect de l'intégrité d'autrui » et avec le style de différents groupes d'usagers de la voie publique (piétons, jeunes, motards, camionneurs, etc.) et qui est compatible avec les habiletés attendues de tout usager en fonction du mode de locomotion utilisé (reconnaissance du signal lumineux, connaissance de sa signification, coordinations oculo-manuelles intactes, techniques nécessaires maitrisées, etc.) et l'état standard du moyen de locomotion utilisé (véhicule avec freins et feux stop en état s'il s'agit d'une automobile).

Cette situation est traduite par le schéma suivant : 


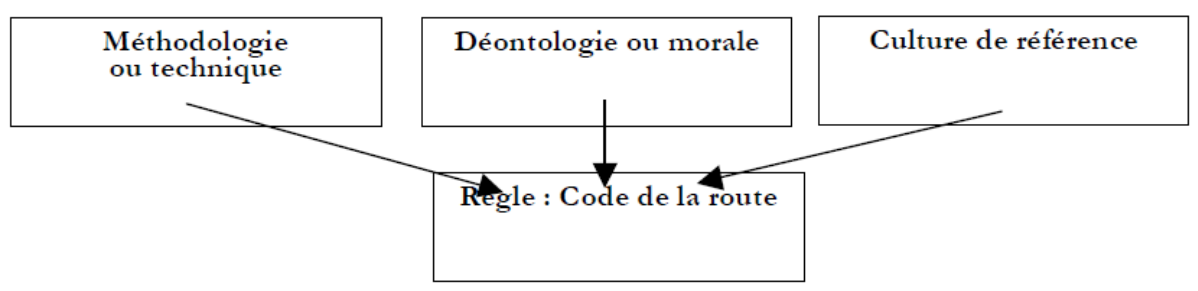

Exemple de divergence ou contradiction des repères

par contre, des cas où les differentes sources de prescription sont conduite de l'usager n'est plus définie par une identité de prescription. Il doit donc se livrer à une délibération et procéder à un choix éthique. Par exemple, l'usager doit opérer un choix entre des sources en conflit (la personne qui transporte un blessé à l'hôpital et qui fait le choix de dépasser la limitation de vitesse, transgresse le code de la route mais est en syntonie avec le précepte moral, qui pose la vie et sa préservation comme principe absolu). Ou bien alors l'usager doit composer une conduite qui ne satisfera à aucune source mais réduira l'intensité du conflit avec chacune d'entre elle (choix du moindre conflit). Ainsi, un automobiliste qui est en retard pour chercher son jeune enfant à l'école, passera au feu orange mais en ayant vérifié qu'aucun piéton n'est prêt à s'engager sur la chaussée. Ce faisant, il récupérera son enfant avec moins de retard que s'il avait attendu le passage au vert (moindre conflit avec la prescription morale, la ponctualité est la politesse des rois) et il enfreindra le code de la route avec discernement, passant certes à l'orange mais sans mettre en danger la vie de qui que ce soit (choix du moindre conflit).

Schéma 3 : situation de divergence entre le code de la route et les autres sources de prescriptions

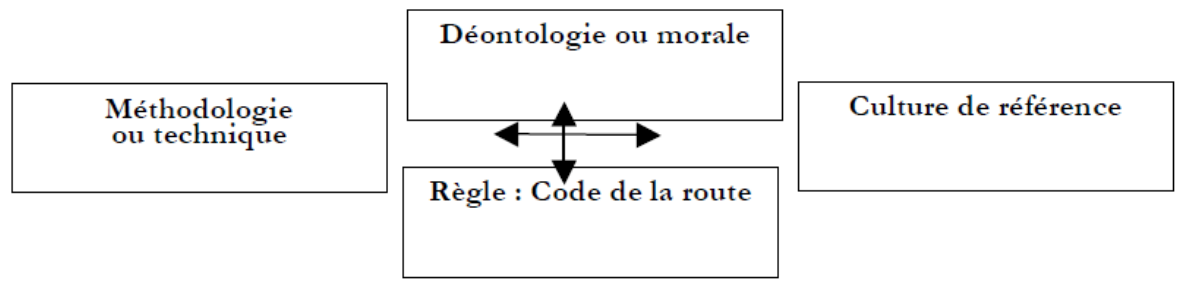

L'utilisation de cette modélisation permet, comme l'a fait Ricoeur pour les conduites en général, de complexifier l'évaluation des situations auxquelles l'usager de la route se trouve confronté parce qu'elle exige de lui une réflexion et une délibération pour arrêter la conduite à tenir. Conduite qui est destinée à préserver l'estime de soi dans un contexte où les directions à suivre sont brouillées du fait de leur multiplicité et de leur noncongruence.

En résumé, le choix éthique consiste à prendre une décision dans le cadre d'une situation qui met en tension des points de vue divergents issus de repères différents. Il peut être 
appréhendé comme une résolution de la crise qu'engendre un « double bind ». En accord avec les propositions de Barel (1989), la résolution s'opère d'au moins deux manières :

- la quête d'un consensus viable (moindre conflit)

- l'adhésion à l'une des repères en conflit.

\section{Prudence des usagers de la voie publique}

Notre modélisation de la délibération a été mise à l'épreuve du terrain que constituent les automobilistes. Par le biais d'un questionnaire spécifique, nous avons cherché à comprendre comment l'usager pouvait effectuer un choix après avoir délibéré, retenir une conduite à tenir. La réponse à des dilemmes nous est apparue comme une méthode intéressante pour appréhender la gestion des zones d'incertitude par les usagers de la voie publique.

Cette méthode est inspirée des travaux de Ricœur sur l'éthique et de Bateson (1980) sur le «double bind». Les dilemmes appellent une personne à choisir entre les deux termes d'une alternative, dont la formule « Boire ou conduire, il faut choisir » est une illustration emblématique. Cet aphorisme nous rappelle que certaines situations exigent du conducteur un raisonnement de type éthique. Le conducteur automobile peut ainsi, dans certaines circonstances, être amené à choisir entre deux façons de se conduire dont aucune n'est pleinement satisfaisante. Nous sommes redevables aux travaux de P. Ricœur sur l'éthique pour la construction des dilemmes.

\subsection{Méthodologie}

Les données seront constituées des réponses fournies à 6 dilemmes par divers types d'usagers de la voie publique. À partir d'un mode déplacement défini, ces dilemmes mettent en balance une attitude conforme au Code de la route avec soit une infraction, soit une prise de risque technique ou une urgence sociale.

$1^{\circ}$ situation - dilemme entre code de la route et santé personnelle - Vous êtes au volant d'une voiture en ville, vous êtes suivi par un gros camion qui peut vous percuter très violemment par l'arrière et vous arrivez sur un feu tricolore qui passe de l'orange au rouge. Vous arrêtez-vous?

$2^{\circ}$ situation - dilemme entre code de la route et secours aux autres - Vous transportez à l'hôpital un ami dont la vie vous semble en danger. Dans une longue ligne droite vous êtes précédé par un véhicule qui respecte la réglementation et roule à $90 \mathrm{~km} /$ heure. Il n'y a personne en face, doublez-vous ce véhicule?

$3^{\circ}$ situation - dilemme entre code de la route et projet professionnel - Vous vous rendez à pied à la gare pour prendre un train et participer à une réunion décisive pour votre avenir professionnel. Vous êtes en retard et vous craignez de manquer ce train. Vous arrivez à un passage piéton qui vient de passer au rouge. Traversez-vous sans attendre?

$4^{\circ}$ situation - dilemme entre code de la route et appartenance au groupe - Vous êtes dans une soirée festive avec des amis. Ils décident soudainement changer de lieu et de s'y rendre en voiture. Ne connaissant pas la route, vous décidez de les suivre. Chemin faisant vous constatez que vous roulez au-delà de la vitesse autorisée. Ralentissez-vous au risque de les perdre? 
Tableau 1 : Les 6 dilemmes

\begin{tabular}{|c|c|c|c|c|c|c|c|}
\hline & & $\begin{array}{l}\text { Dilemme } \\
1\end{array}$ & $\begin{array}{l}\text { Dilemme } \\
2\end{array}$ & $\begin{array}{l}\text { Dilemme } \\
3\end{array}$ & $\begin{array}{l}\text { Dilemme } \\
4\end{array}$ & $\begin{array}{l}\text { Dilemme } \\
5\end{array}$ & $\begin{array}{l}\text { Dilemme } \\
6\end{array}$ \\
\hline Infraction & & $\mathrm{X}$ & $\mathrm{X}$ & $\mathrm{X}$ & & & $\mathrm{X}$ \\
\hline $\begin{array}{l}\text { Prise de risque } \\
\text { technique }\end{array}$ & & & & & $\mathrm{x}$ & $\mathrm{X}$ & \\
\hline \multirow{2}{*}{$\begin{array}{l}\text { Urgence } \\
\text { sociale quant à }\end{array}$} & $\begin{array}{l}\text { laisser } \\
\text { échapper ses } \\
\text { amis }\end{array}$ & & & & $\mathrm{x}$ & & \\
\hline & $\begin{array}{l}\text { manquer un } \\
\text { train au }\end{array}$ & & & $\mathrm{X}$ & & & \\
\hline & $\begin{array}{l}\text { un entretien } \\
\text { d'embauche }\end{array}$ & & & & & $\mathrm{X}$ & \\
\hline & $\begin{array}{l}\text { un passage de } \\
\text { diplôme }\end{array}$ & & & & & & $\mathrm{X}$ \\
\hline & \begin{tabular}{|lr} 
une & blessure \\
au & corps \\
propre &
\end{tabular} & $\mathrm{X}$ & & & & & \\
\hline & la vie d'un ami & & $\mathrm{x}$ & & & & \\
\hline \multirow{3}{*}{$\begin{array}{l}\text { Mode } \\
\text { déplacement }\end{array}$} & pédestre & & & $\mathrm{X}$ & & & \\
\hline & automobile & $x$ & $\mathrm{x}$ & & $\mathrm{X}$ & $\mathrm{X}$ & \\
\hline & bicyclette & & & & & & $\mathrm{X}$ \\
\hline
\end{tabular}

$5^{\circ}$ situation - dilemme entre santé et projet professionnel - Vous allez à un rendezvous extrêmement important pour votre carrière. Vous n'avez pas d'avance sur l'horaire. En route, la neige se met à tomber. Diminuez-vous votre vitesse?

$6^{\circ}$ situation - dilemme entre code de la route et intérêt personnel - Vous êtes pressé car vous allez passer un examen, vous êtes en vélo en ville, tout est bouché, sauf le trottoir. Emprunterez-vous le trottoir pour poursuivre votre déplacement?

Les 6 dilemmes présentés sont structurés comme indiqué dans le tableau suivant:

En fonction des choix effectués pour résoudre les dilemmes, on peut différencier, d'une part des stratégies sécuritaires fondées sur la consonance avec les règles en usage, d'autre part des stratégies prudentes plus ou en moins en dissonance avec ces règles. Le tableau ci-dessous présente les codes utilisés pour repérer les dilemmes. 
Tableau 2 : codes pour repérer les dilemmes

\begin{tabular}{|l|l|l|l|l|l|}
\hline & & $\begin{array}{l}\text { Consonance } \\
\text { à la règle }\end{array}$ & Code & $\begin{array}{l}\text { Dissonance } \\
\text { à la règle }\end{array}$ & Code \\
\hline Dilemme 1 & Le camion & Je freine & D1 C & Je passe & D1 D \\
\hline Dilemme 2 & L'hôpital & Je ne double pas & D2 C & Je double & D2 D \\
\hline Dilemme 3 & Le piéton & J'attends & D3 C & Je traverse & D3 D \\
\hline Dilemme 4 & La sortie & Je ne suis pas & D4 C & J'accélère & D4 D \\
\hline Dilemme 5 & La neige & Je ralentis & D5 C & Je continue & D5 D \\
\hline Dilemme 6 & Le vélo & J'attends & D6 C & Je roule sur le trottoir & D6 D \\
\hline
\end{tabular}

La population interrogée se compose de trois types de conducteurs :

1. les chevronnés: conducteurs ayant plus de sept ans de permis (ils peuvent être des professionnels ou des conducteurs ordinaires);

2. les novices : jeunes titulaires du permis de conduire B ;

3. les apprentis : jeunes n'ayant pas le permis et dont certains sont en formation auto-école, ils ont entre 14 et 18 ans.

\subsection{Analyse des données}

Pour chaque groupe, une première ligne donne les effectifs, la ligne suivante donne les pourcentages.

Tableau 3 : Résultats

\begin{tabular}{|l|l|l|l|l|l|l|l|l|l|l|l|l|}
\hline & D1 C & D1 D & D2 C & D2 D & D3 C & D3 D & D4 C & D4 D & D5 C & D5 D & D6 C & D6 D \\
\hline \multirow{4}{*}{ Chevronnés } & 42 & 54 & 17 & 79 & 54 & 42 & 82 & 14 & 86 & 10 & 30 & 66 \\
\cline { 2 - 12 } & 43,7 & 56,3 & 17,7 & 82,3 & 56,3 & 43,7 & 85,4 & 14,6 & 89,6 & 10,4 & 31,2 & 68,8 \\
\hline \multirow{4}{*}{ Novices } & 11 & 26 & 7 & 30 & 8 & 29 & 34 & 3 & 32 & 5 & 2 & 35 \\
\cline { 2 - 13 } & 29,7 & 70,3 & 18,9 & 81,1 & 21,6 & 78,4 & 91,9 & 8,10 & 86,5 & 13,5 & 5,40 & 94,6 \\
\hline \multirow{3}{*}{ Apprentis } & 41 & 20 & 9 & 52 & 20 & 41 & 51 & 10 & 51 & 10 & 21 & 40 \\
\cline { 2 - 12 } & 67,2 & 32,8 & 14,8 & 85,2 & 32,8 & 67,2 & 83,6 & 16,4 & 83,6 & 16,4 & 34,4 & 65,6 \\
\hline \multirow{2}{*}{ Total } & 94 & 100 & 33 & 161 & 82 & 112 & 167 & 27 & 169 & 25 & 53 & 141 \\
\cline { 2 - 12 } & 48,6 & 51,4 & 17 & 83 & 42,3 & 57,7 & 86,1 & 13,9 & 87,1 & 12,9 & 27,3 & 73,7 \\
\hline
\end{tabular}




\section{a) Des réponses non aléatoires}

57 Nous avons, précédemment, proposé de concevoir l'usager de la voie publique, comme un sujet procédant à des choix. Dans nos réponses aux dilemmes, la possibilité de ne pas choisir peut se manifester sous 3 formes que l'on pourrait qualifier de mécaniques :

1. réponse identique aux 5 dilemmes,

2. réponse alternant un type de choix puis l'autre,

3. réponse manifestant un type de choix pour les 3 premiers dilemmes et le choix opposé pour les trois suivants.

L'examen des réponses montre que ces trois positions sont très marginales, 7 usagers ont répondu identiquement aux 56 dilemmes (choix de conformité), 2 usagers ont alterné leurs choix, 3 usagers ont pris une position pour les 3 premiers dilemmes et la position opposées pour les trois suivants. On peut donc considérer que massivement notre population effectue des choix réfléchis et que l'hypothèse qui considère l'usager de la voie publique comme un acteur prenant des décisions de type éthique est consistante.

\section{b) Des choix qui manifestent de la diversité}

On peut également remarquer, qu'au niveau global, les choix connaissent une certaine diversité :

- les dilemmes 1 et 3 recueillent des réponses qui s'équilibrent entre Consonance avec le Code de la route et dissonance avec celui-ci ;

- Les dilemmes 2 et 6 donnent significativement lieu au choix de la dissonance avec le Code de la route ;

- Les dilemmes 4 et 5 portent des réponses significativement orientées vers la consonance avec le code de la route.

Cette diversité se retrouve aussi dans les différentes populations de l'enquête. Le tableau suivant porte les types dominants :

Tableau 4 : Les types dominants

\begin{tabular}{|l|l|l|l|l|l|l|}
\hline & D1 & D2 & D3 & D4 & D5 & D6 \\
\hline Chevronnés & E & D & E & C & C & D \\
\hline Novices & D & D & D & C & C & D \\
\hline Apprentis & C & D & D & C & C & D \\
\hline Ensemble & E & D & E & C & C & D \\
\hline
\end{tabular}

Légende : E) Equilibre, C) Consonnant, D) Dissonant

61 Ce tableau montre une identité globale de réaction entre les différentes catégories d'usagers et l'ensemble, sauf pour le D3 où les novices se remarquent par une prise de position plus consonante avec le Code de la route. Ce dilemme référant à un conflit entre un rendez-vous d'embauche et le respect du Code de la route, on peut penser que pour la 
population de novices qui la plus concernée par la recherche d'emploi, cette dimension prend un caractère prioritaire voire vital qu'elle ne rencontre pas au sein des deux autres catégories, soit déjà insérées dans le monde du travail, soit encore loin de cette préoccupation.

\section{c) Des choix liés aux types d'usagers}

Si les réponses pointent globalement une réelle diversité, les catégories d'usagers s'opposent peu quant à leur choix majoritaire. Pour autant, on peut se demander s'il est possible de considérer ces réponses comme identiques. Autrement dit se poser la question suivante : le type d'usager a-t-il un effet sur les options retenues pour chaque dilemme?

Pour répondre à cette question, nous avons comparé, à l'aide de la méthode du Chi carré dans sa version comparaison d'échantillons indépendants, les préférences des différents types de populations deux à deux et ce pour chacun des dilemmes présentés.

Tableau 5 : préférences des types d'usager

\begin{tabular}{|c|c|c|c|c|c|}
\hline $\begin{array}{l}\text { D1 } \\
\text { se différentient } \\
\text { des }\end{array}$ & $\begin{array}{ll}\text { D3 } & \\
\text { se } & \text { différentient } \\
\text { des } & \end{array}$ & $\begin{array}{ll}\text { D6 } & \\
\text { se } & \text { différentient } \\
\text { des } & \end{array}$ & & & \\
\hline Chevronnés & Apprentis & Chevronnés & Novices & Chevronnés & Novices \\
\hline Novices & Apprentis & Chevronnés & Apprentis & Novices & Apprentis \\
\hline
\end{tabular}

Ce tableau nous apprend que :

- il n'y a pas de différence significative dans les réponses aux dilemmes D2, D4, D5 (une chance sur cent de commettre une erreur)

- le dilemme 1 discrimine les apprentis des chevronnés et des novices. On peut penser qu'un nombre non négligeable de conducteurs chevronnés et novices ont connu cette expérience. Cela les conduit peut-être à une position plus pragmatique qui consiste à faire peu confiance au conducteur du camion et donc éviter le risque au prix d'une entorse au code de la route.

- Le dilemme 3 sépare les chevronnés des novices et des apprentis. On peut penser que pour la population de conducteurs chevronnés la connaissance du monde travail est plus grande que pour les autres catégories. Elle sait que l'acteur est rarement jugé au plan professionnel sur un seul événement, d'où une relativisation de l'importance de celui-ci.

- Le dilemme 6 différencie d'une part les chevronnés des novices et d'autre part les novices des apprentis. Les différences ne se manifestant pas au plan qualitatif (les trois populations font le même choix) mais au plan quantitatif, il est difficile d'apporter une explication plausible.

\section{d) L'impact de la nature des dilemmes sur les choix}

Enfin, il était légitime de se demander si la nature des dilemmes avait un rôle quant aux choix retenus. Afin d'apporter une réponse à cette interrogation nous avons retenu la méthode du Chi carré dans sa version comparaison d'échantillons appariés.

Le tableau qui suit porte les résultats observés : 
Tableau 6 : Influence de la nature des dilemmes sur les choix retenus

\begin{tabular}{|c|c|c|c|c|c|}
\hline & Dilemme 2 & Dilemme 3 & Dilemme 4 & Dilemme 5 & Dilemme 6 \\
\hline Dilemme 1 & + & & + & + & + \\
\hline Dilemme 2 & & + & + & + & + \\
\hline Dilemme 3 & & & + & + & + \\
\hline Dilemme 4 & & & & & + \\
\hline Dilemme 5 & & & & & + \\
\hline
\end{tabular}

Ce tableau montre que seuls les dilemmes 1 et 3 d'une part et 4 et 5 d'autre part ne sont pas significativement dissemblables. On peut avancer que la proximité des réponses enregistrées pour les dilemmes 1 et 3 est liée au fait que, dans les deux cas, il s'agit d'une opposition entre le code de la route et des éléments importants se référant à la personne (santé propre, avenir professionnel, etc.).

En ce qui concerne les dilemmes 4 et 5 , on peut arguer que la proximité des réponses est liée au fait qu'ils opposent des éléments homogènes. Le dilemme 4 qui confronte le code la route au groupe d'appartenance met ainsi en tension des éléments qui évoquent d'autres acteurs avec lesquels il convient de partager soit la route, soit des plaisirs divers. Le dilemme 5 se situe, au contraire, sur la dimension personnelle, en opposant la santé propre et le projet professionnel.

\section{Conclusion}

En premier lieu, il apparaît que les résultats empiriques relevés, caractérisés par une large hétérogénéité des réponses sur plusieurs plans, confirment la pertinence de l'hypothèse considérant l'usager de la voie publique comme un sujet effectuant des choix éthiques pour conduire. En effet, dans certains cas, celui-ci considère que la conformité aux règles de sécurité du Code de la route ne prime pas sur d'autres impératifs.

En second lieu, le postulat de la défaillance ne saurait être la clef de lecture universelle des manquements au code de la route. L'usager de la voie publique n'est pas forcément quelqu'un qui n'a pas pu ou qui a oublié les règles de sécurité, c'est peut-être aussi quelqu'un qui a voulu exercer son jugement.

71 En troisième lieu, il convient de souligner que le choix de dissonance avec le code de la route est réservé à des situations exceptionnellement graves. On notera que le dilemme 4 , qui n'évoque pas une telle situation; recueille environ $14 \%$ de réponses dissonantes avec les règles du Code de la route.

Enfin, et c'est là une conséquence des points qui précèdent, il conviendrait de revoir la formation des usagers de la voie publique et singulièrement des conducteurs automobiles. La conception actuelle, essentiellement basée sur l'habileté de pilotage et la connaissance $\mathrm{du}$ Code de la route et leur contrôle, ne suffit pas pour ouvrir les routes à des usagers conscients de leur responsabilité. Ces deux piliers de la formation sont adéquats à une 
conception de l'usager comme acteur mû par une recherche de la conformité au Code de la route, sa connaissance liée à l'habileté de maniement des véhicules s'avérant alors suffisante. On est dans le cadre d'une formation centrée sur la sécurité. Celle-ci s'avère insuffisante si l'usager de la route comme est conçu comme une personne prenant des décisions d'ordre éthique lorsque divers impératifs entrent en concurrence. Il s'agit alors, dans ce cas, d'éduquer au jugement et d'aider l'usager à ne jouer avec les règles du code qu'en cas de nécessité absolue, notamment dans les «zones d'incertitude». Dans la mesure ou les « zones d'incertitudes » ne se limitent pas à l'usage de la voie publique, on est enclin à penser qu'il convient de s'orienter vers une éducation du citoyen à la prudence. Cette proposition paraît conforme au regret exprimé par le rapport GADGET qui, après une large étude internationale, déplore que la formation des conducteurs soit trop souvent réduite à des aspects techniques et de réglementation ne sollicitant que des processus intellectuels de bas niveau.

\section{BIBLIOGRAPHIE}

Aristote (1992). Ethique à Nicomaque. Paris : Garnier-Flammarion.

Barel, Y. (1989). Le paradoxe et le système. Grenoble : PUG.

Bateson, G. (1980). Vers une écologie de l'esprit. Tome 2. Paris : Seuil.

Bernoux, P. (1985). La sociologie des organisations. Paris : Edition du Seuil.

Boltanski, L., \& Thévenot J. (1991). De la justification. Paris : Gallimard.

Crozier, M., \& Friedberg E. (1977). L'acteur et le système, Paris : Éditions du Seuil.

Ricoeur, P. (1990), Soi-même comme un autre, Paris : Éditions du Seuil.

Ricoeur, P. (1983), Temps et récit. Tome 1. Paris : Éditions du Seuil.

\section{RÉSUMÉS}

Au regard des statistiques de l'accidentologie, l'usage de la voie publique est un des domaines où la sécurité est un problème majeur. De nombreux travaux consacrés à cette problématique se focalisent davantage sur les outils et méthodes de remédiation que sur une réflexion approfondie du statut de l'usager. Ce dernier apparaît généralement comme un acteur susceptible, voire désireux de se conformer aux règles édictées notamment par le Code de la route. En cas de manquements à l'application des règles conçues pour éviter les accidents, la présomption de défaillance de l'usager s'impose généralement. Sans évacuer l'éventualité de la défaillance, il est possible de concevoir l'usager comme une personne effectuant des choix de natures éthiques. De facto, la notion de sécurité se trouve confrontée, voire remplacée par celle de prudence. Il s'agit alors davantage d'éduquer à la prudence que de former à la sécurité.

Looking at the statistics of accidents, the use of public roads is one of the areas where security is a major issue. Many research papers on this issue focus more on tools and methods than on a 
profound consideration of the status of the user. The latter usually appears as an actor who may, or who is willing to comply with the Highway Code. In case of breaches in the rules designed to prevent accidents, the presumption of fault of the user is typically required. Without removing the possibility of fault, it is possible to designate the user as a person making choices of ethical nature. De facto, the concept of security is facing or replaced by the concept of caution. In this case it is wiser to educate caution than to train security.

INDEX

Mots-clés : sécurité sur la voie publique, accidentologie, éducation à la prudence, formation à la sécurité

Keywords : security on public roads, scientific understanding of accidents, education geared towards caution, training in road security

\section{AUTEUR}

\section{DOMINIQUE VIOLET}

Université de Bordeaux IV 